\title{
Second harmonic Hamiltonian: Algebraic and Schrödinger approaches
}

\author{
T. Mohamadian ${ }^{a}$, H. Panahi ${ }^{a}, *$, J. Negro $^{b}$ \\ a Department of Physics, University of Guilan, Rasht 41635-1914, Iran \\ b Departamento de Física Teórica, Atómica y Óptica, IMUVA, Universidad de Valladolid, 47011 Valladolid, Spain
}

\section{A R T I C L E I N F O}

\section{Article history:}

Received 23 June 2019

Received in revised form 3 October 2019

Accepted 22 October 2019

Available online 28 October 2019

Communicated by B. Malomed

\section{Keywords:}

Second harmonic generation

Bi-confluent Heun equation

Quasi-exactly solvable potential

Polynomial algebra

\begin{abstract}
A B S T R A C T
We study in detail the behavior of the energy spectrum for the second harmonic generation (SHG) and a family of corresponding quasi-exactly solvable Schrödinger potentials labeled by a real parameter $b$. The eigenvalues of this system are obtained by the polynomial deformation of the Lie algebra $s l(2, R)$ representation space. We have found the bi-confluent Heun equation (BHE) corresponding to this system in a differential realization approach, by making use of the symmetries. By means of a $b$-transformation from this second-order equation to a Schrödinger one, we have found a family of quasi-exactly solvable potentials. For each invariant n-dimensional subspace of the second harmonic generation, there are either $n$ potentials, each with one known solution, or one potential with $n$-known solutions. Well-known potentials like a sextic oscillator or that of a quantum dot appear among them.
\end{abstract}

(c) 2019 Elsevier B.V. All rights reserved.

\section{Introduction}

The second harmonic generation (SHG) is a nonlinear optical process in which photons with the frequency of $\omega$ interact with a nonlinear medium and generate photons with twice this frequency [1]. This process was firstly shown when a ruby laser beam passed through the crystal-line quartz and the final beam was twice intense [2]. From classical point of view, the general solution of this system was obtained based on elliptic integrals [3]. The quantum mechanical treatment of this nonlinear optical process is made in Refs. [4-7]. Some authors have considered some non-classical features for this Hamiltonian such as anti-bunching that happened for coherent light incident [8,9], squeezing [10,11], revival-collapse effect in mean photon number of the field modes [12,13], quantum correlation $[14,15]$.

The dynamics of this Hamiltonian can be dealt with in different ways. A semi-classical JWKB analysis is performed for a one dimensional Schrödinger equation and asymptotic formulas are obtained when the input number of photons is large enough [16]. The asymptotic behavior of eigenvalues for large number of input photons and the equivalency between this nonlinear system and one-dimensional Schrödinger with a sextic polynomial potential are shown in Ref. [17]. The exact analytical expression for eigenvalues and eigenstates of this Hamiltonian has been obtained based on the unknown parameter $\lambda[18]$.

\footnotetext{
* Corresponding author.

E-mail addresses: tayebeh.mohamadian.k@gmail.com (T. Mohamadian), t-panahi@guilan.ac.ir (H. Panahi), jnegro@fta.uva.es (J. Negro).
}

The other well-known approach which has been extensively applied in quantum optical system is the polynomial deformation of Lie algebras. These algebras were introduced for the first time by Higgs [19] and Sklyanin [20]. The motivation to apply this method is the existence of nonlinear symmetries in multi-photon Hamiltonian that can not be described by Lie algebras [21]. Furthermore, the general expression for energy spectra of some nonlinear quantum model has been obtained by polynomial deformation of Lie algebra $s u(2)$ in [22]. In [23] the effective Hamiltonian for a nonlinear optical system is found in terms of diagonal operator of polynomial algebra in far-off resonant limit. The SHG system has been considered by the polynomial Lie algebra method in [24] where the authors found an algorithm to achieve eigenvalues. But this algorithmic form could only be applied in some dimensions of a subspace of the system while they had to use the $s u(2)$ quasiclassical approximation in other dimensions. These two methods displayed consistence for some parameters of model but the partial distinctions between them require more enhancements in the approximation method.

The main objective of this paper is to show the general behavior of the energy spectrum for this system by an algebraic approach and to obtain other families of potentials in addition to the sextic potential for this nonlinear optical system within the differential realization approach. In particular we want to illustrate the role of symmetries.

The organization of this paper is as follows. In Section 2 we will use the polynomial deformation of Lie algebra $s l(2, R)$ and its representations to obtain the characteristic equation. By means of a decomposition of the tridiagonal matrix corresponding to the 
Hamiltonian, we can discuss the energy spectrum. We can see that unlike Ref. [16] in which the energy spectra is obtained in the form of convergent power series by a semi-classical approximation, our results can be expressed in an exact manner. In Section 3, the second order partial differential equation of the SHG Hamiltonian will be given through Bargmann-Fock variables and derive an ordinary differential equation, the bi-confluent Heun equation (BHE), by separation in a new set of variables. A further transformation which is characterized by a real label $b$ enables us to pass the form of the BHE into a quasi-solvable Schrödinger equation. The resulting potentials will be parameterized by eigenvalues of the system $E$ and by the transformation label $b$. Some examples are displayed in Section 4. Finally, we will outline the main conclusions at the end of the paper.

\section{Algebraic approach}

The Hamiltonian of the second harmonic generation system with perfect resonance takes the form (in units $\hbar$ ),

$H_{S H G}=\omega a_{1}^{+} a_{1}+2 \omega a_{2}^{+} a_{2}+g\left(a_{1}^{2} a_{2}^{+}+\left(a_{1}^{+}\right)^{2} a_{2}\right)$,

where $a_{1}^{+}\left(a_{1}\right)$ and $a_{2}^{+}\left(a_{2}\right)$ are the creation (annihilation) operators of the input photons with the frequency $\omega$ and the output photon with frequency $2 \omega$, respectively. The parameter $g$ is the coupling constant multiplying a non-diagonal term that describes the interaction between the two coupled bosons. Therefore, the Hamiltonian (2.1) can be rewritten as

$H_{S H G}=H_{0}+g H_{I}$,

showing two basic terms. The role of $H_{0}$ is that of an initial noninteracting Hamiltonian including both type of photons. A set of symmetries (only three of them are functionally independent) of $\mathrm{H}_{0}$ is

$N_{1}=a_{1}^{+} a_{1}, \quad N_{2}=a_{2}^{+} a_{2}, \quad J_{+}=\left(a_{1}^{+}\right)^{2} a_{2}, \quad J_{-}=a_{1}^{2} a_{2}^{+}$.

So, as a two-dimensional Hamiltonian, $H_{0}$ is superintegrable and solvable [25]. The eigenspaces $\mathcal{W}_{n}$ of $H_{0}$ with eigenvalue $n$ are easy to characterize and all of them are finite dimensional (later we will deal with this point). On the other hand, the interaction term of $H_{I}$ is made of the symmetries $J_{+}$and $J_{-}$of $H_{0}$. This is a key point, since it implies that each eigenspace $\mathcal{W}_{n}$ of $H_{0}$ will also be invariant under $H_{I}$. This means that the problem of the diagonalization of the Hamiltonian $H_{S H G}$ can restrict to the family of the finite dimensional eigenspaces of $\mathrm{H}_{0}$.

Once we have introduced the interacting term, the total Hamiltonian $H_{S H G}$ has only one independent additional symmetry given by $H_{0}=\omega\left(N_{1}+2 N_{2}\right)$. Therefore the initial system $H_{0}$ was superintegrable, while the total Hamiltonian $H_{S H G}$ becomes (only) completely integrable $[25,26]$.

In this section, we will write the Hamiltonian in the terms of generators defined by

$J_{+}=\left(a_{1}^{+}\right)^{2} a_{2}, \quad J_{-}=a_{1}^{2} a_{2}^{+}, \quad J_{0}=\frac{a_{1}^{+} a_{1}-a_{2}^{+} a_{2}}{3}$,

$N=a_{1}^{+} a_{1}+2 a_{2}^{+} a_{2}$

as follows:

$H_{S H G}=\omega N+g\left(J_{+}+J_{-}\right)$

The commutators of these generators take the following form

$\left[J_{+}, J_{-}\right]=12 J_{0}^{2}-\frac{N^{2}}{3}-N, \quad\left[J_{0}, J_{ \pm}\right]= \pm J_{ \pm}$,

$\left[N, J_{ \pm}\right]=\left[N, J_{0}\right]=0$
This means that the generators $J_{ \pm}$are lowering/raising with respect to the diagonal operator $J_{0}$, while $N$ is a central generator commuting with all the others. The above set of commutators describes a polynomial deformation of the Lie algebra $\operatorname{sl}(2, \mathbb{R})$. The finite dimensional representation space for these operators in the space spanned by the basis $|J, M\rangle, M=-J, \ldots, J$ is defined by [27]:

$$
\begin{aligned}
J_{0}|J, M\rangle= & (M+c)|J, M\rangle \\
J_{ \pm}|J, M\rangle= & {[(J \mp M)(J \pm M+1)(12 c+4 M \pm 2)]^{\frac{1}{2}} } \\
& \times|J, M \pm 1\rangle,
\end{aligned}
$$

where the parameter $c$ is a real number, $J$ is half integer and the dimension of this space is $2 J+1$. From the commutator $J_{+}$and $J_{-}$and the constraint for finite dimensional space, we have the following relation for $c$ :

$c^{2}=\frac{-J(J+1)}{3}+\frac{1}{12}\left(\frac{n^{2}}{3}+n\right)$,

where $n=n_{1}+2 n_{2}$ is the positive integer value of the generator $N$. For the reality of $c$ in (2.9), we have:

$J \leqslant-\frac{1}{2}+\frac{1}{2}\left(\frac{n^{2}}{3}+n\right)^{\frac{1}{2}}$.

The eigenvalue equation of Hamiltonian (2.1) for a state $\left|\psi_{k}\right\rangle=$ $\sum_{M=-J}^{J} C_{M}^{K}|J, M\rangle$ in an invariant subspace will be

$$
\begin{aligned}
& n \omega C_{M}^{k}+g C_{M-1}^{k}[(J+M)(J-M+1)(12 c+4 M-2)]^{\frac{1}{2}} \\
& \quad+g C_{M+1}^{k}[(J-M)(J+M+1)(12 c+4 M+2)]^{\frac{1}{2}}=E_{k} C_{M}^{k}
\end{aligned}
$$

In matrix notation, the above relation can be written as

$\mathrm{AC}=\mathbf{0}$

i.e., the product of a tridiagonal symmetric matrix denoted $\mathbf{A}$ of dimensions $\hat{n} \times \hat{n}$ by a column matrix $\mathbf{C}$ of the coefficients $C_{M}^{K}$. The elements of main diagonal are $n \omega-E_{K}$ and the elements of the other two diagonals are the coefficients of $C_{M \pm 1}^{K}$ which will be called $a_{i}, i=1, \ldots, \hat{n}-1$. Hence, the eigenvalues $E_{K}$ will be found from the determinant of this tridiagonal matrix.

We can face this problem under different points of view. One of them is that used in Ref. [28]. In this paper, this type of matrix is decomposed to two matrices. One of them is a lower triangular matrix where all elements of the main diagonal are equal to 1 and the other one is an upper triangular matrix. The determinant of tridiagonal matrix will be product of the determinant of these matrices which gives the product of the elements of main diagonal of the second one. In this way, we have the following relation for the determinant of $A$ :

$$
\begin{aligned}
& \operatorname{det}(A)=(-\lambda)\left(-\lambda-\frac{a_{1}^{2}}{-\lambda}\right)\left(-\lambda-\frac{a_{2}^{2}}{-\lambda-\frac{a_{1}^{2}}{-\lambda}}\right) \ldots \\
& \left(-\lambda-\frac{a_{\hat{n}}^{2}}{-\lambda-\frac{a_{\hat{n}-1}^{2}}{\frac{\cdots}{-\lambda-\frac{a_{1}^{2}}{-\lambda}}}}\right)
\end{aligned}
$$

where $-\lambda=n \omega-E_{K}$. We can also write this relation as follows, 
$\operatorname{det}(A)=(-\lambda)\left(\delta-\frac{\gamma_{1}}{\delta}\right)\left(\delta-\frac{\gamma_{2}}{\delta-\frac{\gamma_{1}}{\delta}}\right) \ldots\left(\delta-\frac{\gamma_{\hat{n}}}{\delta-\frac{\gamma_{\hat{n}-1}}{\frac{\cdots}{\delta-\frac{\gamma_{1}^{2}}{\delta}}}}\right)$

with $\delta=-\lambda$ and $\gamma_{i}=a_{i}^{2}$. Next, we can see the recurrence relation for the determinant by using the following definition:

$d_{0}=1$,

$d_{1}=\delta$,

$d_{2}=\delta d_{1}-\gamma_{1}$,

$d_{3}=\delta d_{2}-\gamma_{2} d_{1}$,

$d_{4}=\delta d_{3}-\gamma_{3} d_{2}$,

$:=$

$d_{\hat{n}}=\delta d_{\hat{n}-1}-\gamma_{\hat{n}-1} d_{\hat{n}-2}$.

In this way, the $\operatorname{det}(A)$ will be

$$
\begin{aligned}
\operatorname{det}(A)= & d_{1} \times\left(\frac{d_{2}}{d_{1}}\right) \times\left(\frac{d_{3}}{d_{2}}\right) \times\left(\frac{d_{4}}{d_{3}}\right) \times \cdots \times\left(\frac{d_{\tilde{n}-1}}{d_{\hat{n}-2}}\right) \\
& \times\left(\frac{d_{\hat{n}}}{d_{\hat{n}-1}}\right),
\end{aligned}
$$

then, the condition $\operatorname{det}(A)=0$ can be expressed as

$d_{\hat{n}}=\delta d_{\hat{n}-1}-\gamma_{\hat{n}-1} d_{\hat{n}-2}=0$.

By means of Eq. (2.17), we can discuss the eigenvalues of the system. The above recurrence relation for Hamiltonian (2.1) was obtained in [18] but there it was assumed that the form of eigenvalues of this system as $E=\omega N+g \lambda$ with the unknown parameter $\lambda$ and then it was found the recurrence relation for $\lambda$. In our approach, all elements in (2.17) are known and they are related to parameters of the system.

\subsection{The finite dimensional subspace $\mathcal{W}_{n}$}

In order to discuss about the eigenvalues of this system, we have to characterize the eigenspace $\mathcal{W}_{n}$ of $H_{0}$ with eigenvalue $n$. For this aim, we change our basis from $|J, M\rangle$ to the basis of twomode Fock space $\left|n_{1}, n_{2}\right\rangle$ with two constrains $n=n_{1}+2 n_{2}$ and $M+c=\frac{n_{1}-n_{2}}{3}$. The subspaces $\mathcal{W}_{n}$ have the two following features:

- The subspaces $\mathcal{W}_{n}$ are finite-dimensional i.e., for each $N=n$ photon number, a basis will be formed by the following $\left[\frac{n}{2}\right]+1$ vectors,

$|n, 0\rangle,|n-2,1\rangle,|n-4,2\rangle, \cdots,\left|n-2\left[\frac{n}{2}\right],\left[\frac{n}{2}\right]\right\rangle$.

So, the dimension of this subspace for the odd and even values $n=2 k$ and $n=2 k+1$ is equal to $\hat{n}=k+1$.

- The subspaces $\mathcal{W}_{n}$ are invariant under $H$ and its restriction to these invariant subspaces result in the finite dimensional matrix representation for $H$ denoted as $H_{n}$. In this way, a portion of the spectrum of $H$ can be obtained by diagonalizing the matrix $H_{n}$. According to the definition in [29] we can use the term "quasiexactly solvable" for the second harmonic generation system.
Now we have two situations for this system:

a) Even dimension $\hat{n}=2 \tilde{k}, \tilde{k} \in \mathbb{N}$

In this case, the determinant $d_{\hat{n}}$ has the general form

$$
\begin{aligned}
d_{2 \tilde{k}} & =\delta^{2 \tilde{k}}+b_{1} \delta^{2 \tilde{k}-2}+b_{2} \delta^{2 \tilde{k}-4}+\cdots+b_{n} \delta^{2}+b_{0}=0 \\
& \Longrightarrow \delta= \pm \sqrt{\xi_{i}},
\end{aligned}
$$

where $\xi_{i}$ is a combination of $b_{i}$ 's $i=(1,2, \ldots, n)$ and $b_{i}$ 's are used for different combination of $a_{i}$ 's. When the dimension of the system is even, the behavior of the eigenvalues will take the form $E=E_{0} \pm \sqrt{\xi_{i}}$. Also, let us mention that when the number of photons $n$ is 18 this means that the degree of the polynomial equation (2.19) in terms of $\delta^{2}$ is 5. Based on AbelRuffini theorem, we can not find radical expressions for the roots of any polynomial equation with degree more than five [30], hence for $n \geq 18$ we may not have algebraic solutions.

b) Odd dimension $\hat{n}=2 \tilde{k}+1, \tilde{k} \in \mathbb{N}$.

In this case $d_{\hat{n}}$ will have the form

$$
\begin{aligned}
& d_{2 \tilde{k}+1}=\delta^{2 \tilde{k}+1}+b_{1} \delta^{2 \tilde{k}-1}+b_{2} \delta^{2 \tilde{k}-3}+\cdots+b_{n} \delta=0 \\
& \Longrightarrow \delta=0, \quad \delta= \pm \sqrt{\xi_{i}} .
\end{aligned}
$$

So, when the dimension of the system is odd, it is seen that one of the roots for equation (2.20) will be zero $\delta=0$, and its corresponding eigenvalue will be $E_{k}=E_{0}=n \omega$.

As an example, we can see this behavior in two cases $n=2$ and $n=4$.

1) $n=2$

The two-dimensional Fock subspace $\mathcal{W}_{2}$ has the basis $\{|2,0\rangle$, $|0,1\rangle\}$. The Hamiltonian restricted to this subspace in this basis has the matrix form

$H_{2}=\left(\begin{array}{cc}2 \omega & g \sqrt{2} \\ g \sqrt{2} & 2 \omega\end{array}\right)$.

The eigenvalue equation will be

$\left(2 \omega-E_{k}\right)^{2}-2 g^{2}=0$,

whose eigenvalues $E_{k}$ are

$E_{k}=2 \omega \pm \sqrt{2} g$.

2) $n=4$

The subspace $\mathcal{W}_{4}$ is generated by the vectors $|4,0\rangle,|2,1\rangle$, $|0,2\rangle$. The matrix representation in this subspace will be

$H_{4}=\left(\begin{array}{ccc}4 \omega & 2 g \sqrt{3} & 0 \\ 2 g \sqrt{3} & 4 \omega & 2 g \\ 0 & 2 g & 4 \omega\end{array}\right)$.

From the following eigenvalue equation

$\left(4 \omega-E_{k}\right)^{3}-16 g^{2}\left(4 \omega-E_{k}\right)=0$.

The eigenvalues from this equation will be $E_{1}=4 \omega+4 g, E_{2}=$ $4 \omega-4 g, E_{3}=4 \omega$. As we can see, one of the eigenvalues $\left(E_{3}\right)$ is in the form $n \omega$.

\section{The associated Schrödinger equations}

\subsection{Differential realizations of the second harmonic generation model}

The Hamiltonian (2.1) in the Bargmann-Fock representation by using following expression of creation and annihilation operators 
$a_{1}=\partial_{z_{1}}, \quad a_{1}^{+}=z_{1}, \quad a_{2}=\partial_{z_{2}}, \quad a_{2}^{+}=z_{2}$,

adopts the differential form

$H_{S H G}=g z_{2} \partial_{z_{1}}^{2}+g z_{1}^{2} \partial_{z_{2}}+2 z_{2} \partial_{z_{2}}+z_{1} \partial_{z_{1}}, \quad \omega=1$.

As we mentioned in the previous section, this system has a set of two independent and commuting symmetry operators, including the Hamiltonian $\mathrm{H}_{S H G}$,

$\left\{H_{S H G}, H_{0}=\omega\left(N_{1}+2 N_{2}\right)\right\}$.

The symmetry $H_{0}$ will be a first order differential operator in the Bargmann-Fock representation

$H_{0}=z_{1} \partial_{z_{1}}+2 z_{2} \partial_{z_{2}}, \quad \omega=1$.

In this realization, the Fock states are represented by the monomials

$\left|n_{1}, n_{2}\right\rangle=\frac{\left(z_{1}\right)^{n_{1}}\left(z_{2}\right)^{n_{2}}}{\left(n_{1} ! n_{2} !\right)^{1 / 2}}$.

The subspace $\mathcal{W}_{n}$ will be generated by the above monomials such that $n_{1}+2 n_{2}=n=E_{0}$ (we suppose $\omega=1$ ). The symmetry operator $H_{0}$ will help us to make a transformation from variables $\left(z_{1}, z_{2}\right)$ to $(s, t)$ so that the Hamiltonian $H$ can be made separable. We can find this transformation by imposing that $H_{0}$ depends only on one variable. After straightforward calculations, we arrive at the following (non unique) simple solution:

$s=z_{2}, \quad t=\frac{z_{1}^{2}}{z_{2}}$.

We can express the partial derivatives $\frac{\partial}{\partial z_{1}}$ and $\frac{\partial}{\partial z_{2}}$ in terms of new variables as follows,

$\frac{\partial}{\partial z_{1}}=2 \sqrt{\frac{t}{s}} \frac{\partial}{\partial t}$

$\frac{\partial}{\partial z_{2}}=\frac{\partial}{\partial s}-\frac{t}{s} \frac{\partial}{\partial t}$.

The Hamiltonians $H_{S H G}$ and $H_{0}$ in the new variables will have the following expression

$H_{S H G}=4 g t \frac{\partial^{2}}{\partial t^{2}}+g\left(2-t^{2}\right) \frac{\partial}{\partial t}+g t s \frac{\partial}{\partial s}+2 s \frac{\partial}{\partial s}$,

$H_{0}=2 s \frac{\partial}{\partial s}$.

The simultaneous eigenfunctions for $H_{S H G}$ and $H_{0}$ represented as $\Psi(s, t)$ satisfy,

$H_{S H G} \Psi(s, t)=E \Psi(s, t)$

$H_{0} \Psi(s, t)=E_{0} \Psi(s, t) \Longrightarrow \Psi(s, t)=s^{\frac{E_{0}}{2}} \psi(t)$,

where $E_{0}=n$ is determined by the eigenspace $\mathcal{W}_{n}$ of $H_{0}$ in which we are restricting the Hamiltonian $H$. Replacing this separated eigenfunctions in the eigenvalue equation for $H_{S H G}$ in (3.9), we get a second-order reduced differential equation for $\psi(t)$ :

$4 g t \frac{d^{2} \psi}{d t^{2}}+g\left(2-t^{2}\right) \frac{d \psi}{d t}+\left(E_{0}-E+\frac{g t E_{0}}{2}\right) \psi=0$.

This equation can be related to the bi-confluent Heun equation (BHE) because it has two singularities, at the origin and at the infinity, the first one being regular and the second one irregular [31]. The standard bi-confluent Heun equation has the following form

$$
\begin{aligned}
& x\left(\frac{d^{2} y}{d x^{2}}\right)+\left(1+\alpha+\beta x-2 x^{2}\right)\left(\frac{d y}{d x}\right) \\
& \quad+\left(-\frac{\delta+(1+\alpha) \beta}{2}+(\gamma-\alpha-2) x\right) y=0 .
\end{aligned}
$$

In order to get this form we make a new change of variable $t=$ $m \rho$, so we can rewrite the eigenvalue equation (3.12) as follows

$$
\begin{aligned}
& \rho\left(\frac{d^{2} \psi}{d \rho^{2}}\right)+\left(\frac{1}{2}-\frac{m^{2} \rho^{2}}{4}\right)\left(\frac{d \psi}{d \rho}\right) \\
& +\left(\frac{\left(E_{0}-E\right) m}{4 g}+\frac{E_{0} m^{2} \rho}{8}\right) \psi(\rho)=0 .
\end{aligned}
$$

Then, if we choose $m^{2}=8$ we will have that (3.14) becomes:

$\rho \frac{d^{2} \psi}{d \rho^{2}}+\left(\frac{1}{2}-2 \rho^{2}\right) \frac{d \psi}{d \rho}+\left(\frac{m\left(E_{0}-E\right)}{4 g}+E_{0} \rho\right) \psi=0$,

which is exactly in the form of (3.13) with the following identification of parameters:

$\alpha=-\frac{1}{2}, \quad \beta=0, \quad \gamma=E_{0}+\frac{3}{2}, \quad \delta=-\frac{m\left(E-E_{0}\right)}{2 g}$.

If we apply power series (Frobenius) method to Eq. (3.15) with $\psi(\rho)$ in the form $\sum_{i=0}^{\infty} \tilde{b}_{i}(\rho)^{i+\kappa}$, we will find two sets of solutions for $\kappa=0$ and $\kappa=1 / 2$ in following form:

$\psi(\rho)=\sum_{i=0}^{\infty} \tilde{b}_{i} \rho^{i} \quad$ for $\quad \kappa=0$,

$\psi(\rho)=\sum_{i=0}^{\infty} \tilde{b}_{i} \rho^{i+1 / 2}$ for $\quad \kappa=1 / 2$.

Then, the wavefunctions $\Psi$ in the old variables $z_{1}, z_{2}$ will be

$\Psi\left(z_{1}, z_{2}\right)=\sum_{i=0}^{\infty} \tilde{b_{i}}\left(z_{1}\right)^{2 i}\left(z_{2}\right)^{\frac{E_{0}}{2}-i} \quad$ for $\quad \kappa=0$,

$\Psi\left(z_{1}, z_{2}\right)=\sum_{i=0}^{\infty} \tilde{b}_{i}\left(z_{1}\right)^{2 i+1}\left(z_{2}\right)^{\frac{E_{0}}{2}-i-\frac{1}{2}}$ for $\kappa=1 / 2$.

From equation (3.5), we can see that the power of $z_{2}$ has to be integer. So we consider $E_{0}=2 k$ in (3.19) and $E_{0}=2 k+1$ in (3.20). Then we have the following relation

$\Psi\left(z_{1}, z_{2}\right)=\sum_{i=0}^{\infty} \tilde{b}_{i}\left(z_{1}\right)^{2 i}\left(z_{2}\right)^{k-i} \quad$ for $\quad E_{0}=2 k$,

$\Psi\left(z_{1}, z_{2}\right)=\sum_{i=0}^{\infty} \tilde{b}_{i}\left(z_{1}\right)^{2 i+1}\left(z_{2}\right)^{k-i}$ for $E_{0}=2 k+1$.

As we see, the function $\Psi\left(z_{1}, z_{2}\right)$ will have a singularity for $i>$ $k$ in both cases. Then, in order $\Psi\left(z_{1}, z_{2}\right)$ to be an analytical and polynomial solution, the power of $z_{2}$ must be greater or equal than zero and the upper bound in (3.21) and (3.22) power series has to be $k$. If we write $\Psi\left(z_{1}, z_{2}\right)$ in new variables $(s, \rho)$, the function $\Psi(s, \rho)$ will be

$$
\begin{array}{ll}
\Psi(s, \rho)=(s)^{k} \sum_{i=0}^{k} \tilde{b}_{i} m^{i} \rho^{i}, & E_{0}=2 k, \\
\Psi(s, \rho)=(s)^{k+\frac{1}{2}} \sum_{i=0}^{k} \tilde{b}_{i} m^{i+\frac{1}{2}} \rho^{i+1 / 2}, & E_{0}=2 k+1,
\end{array}
$$


with

$\psi(\rho)=\sum_{i=0}^{k} \tilde{b}_{i}(m \rho)^{i}, \quad E_{0}=2 k$,

$\psi(\rho)=\sum_{i=0}^{k} \tilde{b}_{i}(m \rho)^{i+\frac{1}{2}}, \quad E_{0}=2 k+1$.

\subsection{QES Schrödinger potentials}

Every second-order second order equation of the form (3.15) can be transformed into a Schrödinger equation by means of a suitable change of variables and a gauge transformation [29]. We consider the case $m=2 \sqrt{2}$ (we will discuss the other sign later) and use the similarity transformation and the change of variables given by

$\psi(\rho)=\exp [-W(x)] \chi(x), \quad x=\chi(\rho)$.

Then, if the function $W(x)$ and the variable $x(\rho)$ satisfy the following equation:

$\ddot{x}-2(\dot{x})^{2} \frac{d W}{d x}+\dot{x}\left(\frac{1}{2 \rho}-2 \rho\right)=0$,

the function $\chi(\rho)$ will satisfy an Schrödinger equation (without the first order derivative) of the form

$-\chi^{\prime \prime}+V(x) \chi=\lambda \chi$,

where $\dot{x}=\frac{d x}{d \rho}$ and the expression for the potential will be

$$
\begin{aligned}
V(x)= & \frac{\ddot{x}}{\dot{x}^{2}} \frac{d W}{d x}-\left(\frac{d W}{d x}\right)^{2}+\frac{d^{2} W}{d x^{2}}+\frac{1}{\dot{x}} \frac{d W}{d x}\left[\frac{1}{2 \rho}-2 \rho\right] \\
& -\frac{a_{0}+\rho E_{0}}{\rho \dot{x}^{2}},
\end{aligned}
$$

where $a_{0}$ is

$a_{0}=\frac{\sqrt{2}\left(E_{0}-E\right)}{2 g}$.

We can find different forms for the function $W(x)$ and the potential $V(x)$ based on the new variable $x(\rho)$. A simple choice of this function is just a power $x(\rho)=\rho^{b}$, with $b \in \mathbb{R}^{+}$. In this case we get the following expressions for $V(x)$ and $\chi(x)$ :

$$
\begin{aligned}
V_{b}(x)= & \left(-\frac{1}{4}+\frac{1}{16 b^{2}}\right) x^{-2}-\left(\frac{a_{0}}{b^{2}}\right)(x)^{-2+\frac{1}{b}} \\
& -\left(\frac{3+2 E_{0}}{2 b^{2}}\right)(x)^{-2+\frac{2}{b}}+\frac{1}{b^{2}}(x)^{-2+\frac{4}{b}}, \\
\chi_{b}(x)= & x^{-(1-2 b) /(4 b)} \exp \left[-\frac{x^{\frac{2}{b}}}{2}\right] \psi(\rho(x)) .
\end{aligned}
$$

The wavefunction $\chi$ in (3.33) will be physical square integrable for $b \geqslant \frac{1}{2}$, assuming that $\psi(\rho)$ is a polynomial. We can get different realizations of this potential for the following values of $b: 1 / 2,1$, and 2 . In this way we obtain some simplified expressions by the vanishing of one of the powers of $x$ in (3.32).

1) $b=1 / 2$.

For this value of $b$, the potential and wavefunction respectively will be
$V_{1 / 2}(x)=4 x^{6}-x^{2}\left(6+4 E_{0}\right)-4 a_{0}$,

$\chi_{\frac{1}{2}}(x)=\exp \left[-\frac{x^{4}}{2}\right] \psi(\rho(x))$.

According to the definition of $a_{0}$ in (3.31), we can replace

$a_{0}=\frac{\sqrt{2} E_{0}}{2 g}-\frac{\sqrt{2}}{2 g} E$,

and redefine

$\tilde{V}_{1 / 2}(x)=4 x^{6}-x^{2}\left(6+4 E_{0}\right)-\frac{2 \sqrt{2} E_{0}}{g}$.

Then, the resulting Schrödinger equation is:

$-\chi_{1 / 2}^{\prime \prime}(x)+\tilde{V}_{1 / 2}(x) \chi_{1 / 2}(x)=-\frac{2 \sqrt{2}}{g} E \chi_{1 / 2}(x)$.

Therefore in this case we have a sextic potential depending on the parameter $E_{0}$ of the finite dimensional eigenspace while the energy $\lambda$ for $\chi_{1 / 2}(x)$ correspond to the values of $(-2 \sqrt{2} E) / g$ in this eigenspace. The potential $\tilde{V}_{1 / 2}(x)$ depends on $x^{2}$ and wave function $\chi_{1 / 2}(x)$ depends also on polynomials in $x^{2}$. This means that this potential is symmetric in $x$ and the even and odd solutions can be obtained from (3.35) with (3.25) and (3.26) respectively for even and odd number of photons.

2) $b=1$.

In this case the potential function is

$V_{1}(x)=-\frac{3}{2}-\frac{3}{16 x^{2}}+x^{2}-\frac{a_{0}}{x}-E_{0}$.

We can discuss about this potential in two cases $a_{0}=0$ and $a_{0} \neq 0$.

When the dimension of the eigenspace is odd, then according to the odd case we mentioned in Section 2.1, the parameter $a_{0}$ will be zero for one of the eigenvalues $\left(E_{k}=E_{0}\right)$. So we can consider the potential $\tilde{V}_{1}(x)$ for this case as an oscillator potential, plus a centrifugal term, with energy $E_{0}$. The potential $\tilde{V}_{1}(x)$ is

$\tilde{V}_{1}(x)=-\frac{3}{2}-\frac{3}{16 x^{2}}+x^{2}$.

In other cases $\left(a_{0} \neq 0\right)$, there will coexist an oscillator and centrifugal plus a Coulomb potential. This potential as

$\tilde{\tilde{V}}_{1}(x)=-\frac{3}{2}-\frac{3}{16 x^{2}}+x^{2}-\frac{a_{0}}{x}$,

can be obtained from a system of two interacting electrons in an external harmonic potential. Examples of this kind of system are given as quantum dots, and it is mentioned in many references, see for example [32-34].

The energy $\lambda$ of the Schrödinger equation for both cases is $E_{0}$ and the corresponding wavefunction $\chi_{1}(x)$ is

$\chi_{1}(x)=x^{\frac{1}{4}} \exp \left[-\frac{x^{2}}{2}\right] \psi(\rho(x))$.

3) $b=2$.

In this case, the potential is

$V_{2}(x)=\frac{1}{4}-\frac{15}{64 x^{2}}-\frac{a_{0}}{4 x^{3 / 2}}+\frac{-3-2 E_{0}}{8 x}$.

In this case, the potential includes as intrinsic parameters both $a_{0}$ and $E_{0}$, which can not take the role of a non-vanishing energy, thus the energy of the corresponding Schrödinger equation is zero: 

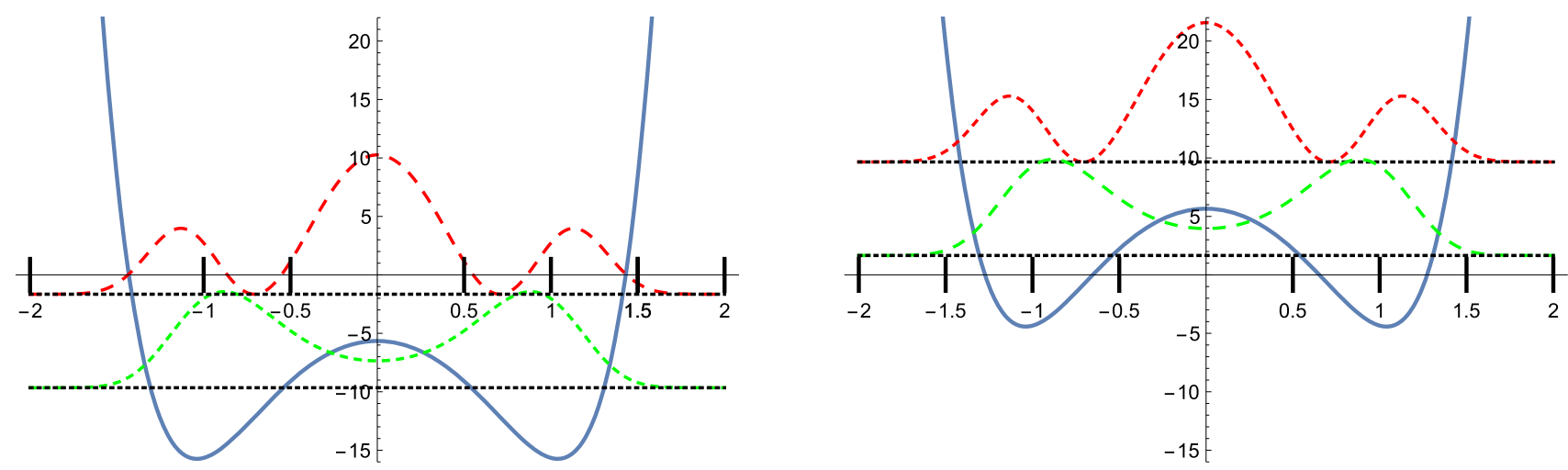

Fig. 1. The potential $\tilde{V}_{1 / 2}(x)$ (solid line) and probability density function $\left|\chi_{p}(x)\right|^{2}$ (dashed line) for $n=2$ in cases $t=2 \sqrt{2} \rho$ placed at the energy levels (dotted lines) $\varepsilon_{ \pm}=-2 \sqrt{2}\left(2 \pm \sqrt{2}\right.$ ) (left) and $t=-2 \sqrt{2} \rho$ placed at the energy levels (dotted lines) $\varepsilon_{ \pm}=2 \sqrt{2}(2 \pm \sqrt{2}$ ) (right).

$$
-\chi_{2}^{\prime \prime}(x)+V_{2}(x) \chi_{2}(x)=0
$$

The wavefunction $\chi_{2}(x)$ has the expression

$$
\chi_{2}(x)=x^{\frac{3}{8}} \exp \left[-\frac{x}{2}\right] \psi(\rho(x)) \text {. }
$$

If we replace $\rho$ by $-\rho$ in (3.15), we will have the same differential equation but for a negative value $m=-2 \sqrt{2}$. Hence we encounter another set of potentials in which the relation between $x$ and $\rho$ are in the form $x=(-\rho)^{b}$ rather than $x=(\rho)^{b}$.

\section{Two examples: the sextic and the quantum dot potentials}

As we have seen in the last section, potentials $\tilde{V}_{1}(x), \tilde{\tilde{V}}_{1}(x)$ and $\tilde{V}_{1 / 2}(x)$ represent well known physical system. In this section, we concentrate on the illustration of these potentials and corresponding probability density functions for (i) two-dimensional $\left(\mathcal{W}_{2}\right)$ and (ii) three-dimensional $\left(\mathcal{W}_{3}\right)$ subspaces. The examples of these two subspaces were discussed in Section 2 . The potentials $\tilde{\tilde{V}}_{1}(x)$ for $n=2$ and $\tilde{V}_{1 / 2}(x)$ for $n=2$ and $n=3$ are considered in twodimensional subspace. In three-dimensional subspace, the potentials $\tilde{\tilde{V}}_{1}(x)$ and $\tilde{V}_{1}(x)$ are illustrated for $n=4$ as well the even and odd solutions for the potential $\tilde{V}_{1 / 2}(x)$ are shown for $n=4$ and $n=5$ respectively.

\subsection{Two-dimensional subspace $\mathcal{W}_{2}$}

The bases and matrix representation for two-dimensional subspace of $n=2$ was obtained as an example in Section 2. If we choose $\omega=1$ and $g=1$ for simplicity, the eigenvalues and eigenfunctions corresponding to the two by two matrix Hamiltonian are

$$
\left|\psi_{p}\right\rangle=\gamma_{p, 1}|2,0\rangle+\gamma_{p, 2}|0,1\rangle, \quad p=1,2,
$$

with

\begin{tabular}{|r|c|r|r|}
\hline$p$ & $E_{p}$ & $\gamma_{p, 1}$ & $\gamma_{p, 2}$ \\
\hline 1 & $2+\sqrt{2}$ & $1 / \sqrt{2}$ & $1 / \sqrt{2}$ \\
\hline 2 & $2-\sqrt{2}$ & $1 / \sqrt{2}$ & $-1 / \sqrt{2}$ \\
\hline
\end{tabular}

We can write the eigenfunctions (4.1) in terms of the variables $\rho, s$ (see (3.25)) as follows:

$\Psi_{p}(\rho, s)=s\left(\gamma_{p, 2}+2 \gamma_{p, 1} \rho\right)=s \psi(\rho)$.

The wavefunctions are

$\chi_{p}(x)=e^{W(x)}\left(\gamma_{p, 2}+2 \gamma_{p, 1} \rho\right)$.
When we choose $b=1 / 2$, that is $x=\sqrt{\rho}$, we have the Schrödinger equation with potential $\tilde{V}_{1 / 2}(x)$ as (3.37) with the energy $\varepsilon_{ \pm}=$ $-2 \sqrt{2}(2 \pm \sqrt{2})$. The potential $\tilde{V}_{1 / 2}(x)$ and the probability density function $\left|\chi_{p}(x)\right|^{2}$ are depicted in Fig. 1 (left). The corresponding potential and density $\left|\chi_{p}(x)\right|^{2}$ are shown for the case $t=-2 \sqrt{2} \rho$ with the energy $\varepsilon_{ \pm}=2 \sqrt{2}(2 \pm \sqrt{2})$ in Fig. 1 (right), in this case the new potential is the same as before except for the addition of a constant (see Fig. 1 (right)). As we can see, the potential $\tilde{V}_{1 / 2}(x)$ is symmetric in $x$ and the solutions here obtained are even solutions.

If we want to show the odd solutions for the potential $\tilde{V}_{1 / 2}(x)$, we have to find the wavefunctions for $n=3$. The bases of twodimensional subspace are $\{|3,0\rangle,|1,1\rangle\}$ for this case. The corresponding two by two Hamiltonian in this subspace has the following eigenfunctions and eigenvalues

$\left|\psi_{p}\right\rangle=\gamma_{p, 1}|3,0\rangle+\gamma_{p, 2}|1,1\rangle, \quad p=1,2$,

with

\begin{tabular}{|r|c|r|r|}
\hline$p$ & $E_{p}$ & $\gamma_{p, 1}$ & $\gamma_{p, 2}$ \\
\hline 1 & $3+\sqrt{6}$ & $1 / \sqrt{2}$ & $1 / \sqrt{2}$ \\
\hline 2 & $3-\sqrt{6}$ & $1 / \sqrt{2}$ & $-1 / \sqrt{2}$ \\
\hline
\end{tabular}

The eigenfunctions (4.4) can be written as a function with variables $\rho, s$ (see (3.26)) as follows:

$\Psi_{p}(\rho, s)=s^{\frac{3}{2}}\left(\gamma_{p, 2} \rho^{\frac{1}{2}}+\frac{2}{\sqrt{3}} \gamma_{p, 1} \rho^{\frac{3}{2}}\right)=s \psi(\rho)$.

The wavefunctions are

$\chi_{p}(x)=e^{W(x)}\left(\gamma_{p, 2} x+\frac{2}{\sqrt{3}} \gamma_{p, 1} x^{3}\right)$.

The $\tilde{V}_{1 / 2}(x)$ and corresponding probability density functions $\left|\chi_{p}(x)\right|^{2}$ are depicted in Fig. 2 for $t= \pm 2 \sqrt{2} \rho$ with corresponding energies.

For another case $b=1$, that is, $x(\rho)=\rho$, we face potential $\tilde{\tilde{V}}_{1}(x)$. The corresponding potential and the probability density function $\left|\chi_{p}(x)\right|^{2}$ are shown for $t=-2 \sqrt{2} \rho$ in Fig. 3 and $t=-2 \sqrt{2} \rho$ in Fig. 4. In this case the potentials for different signs are simply interchanged.

\subsection{Three-dimensional subspace $\mathcal{W}_{3}$}

For $n=4$, we encounter a three dimensional subspace as we mentioned in Section 2.1 where the Hamiltonian has a $3 \times 3$ matrix representation. The eigenvalues and eigenfunctions in this subspace are 

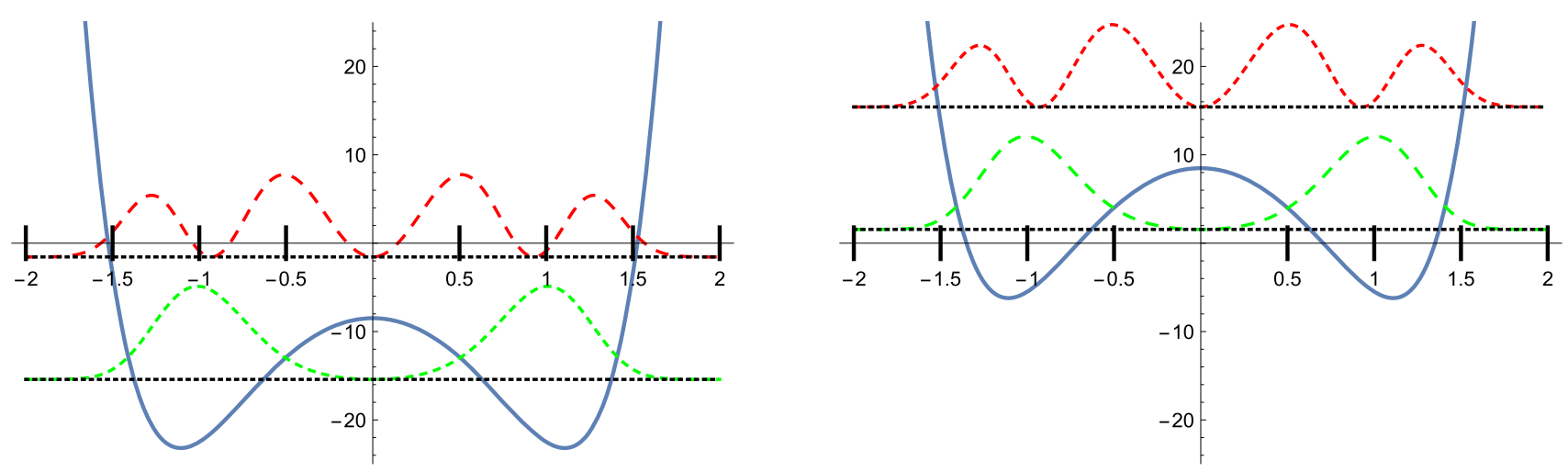

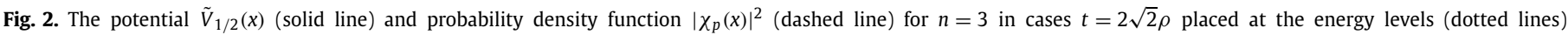
$\varepsilon_{ \pm}=-2 \sqrt{2}(2 \pm \sqrt{2})$ (left) and $t=-2 \sqrt{2} \rho$ placed at the energy levels (dotted lines) $\varepsilon_{ \pm}=2 \sqrt{2}(2 \pm \sqrt{2})$ (right).
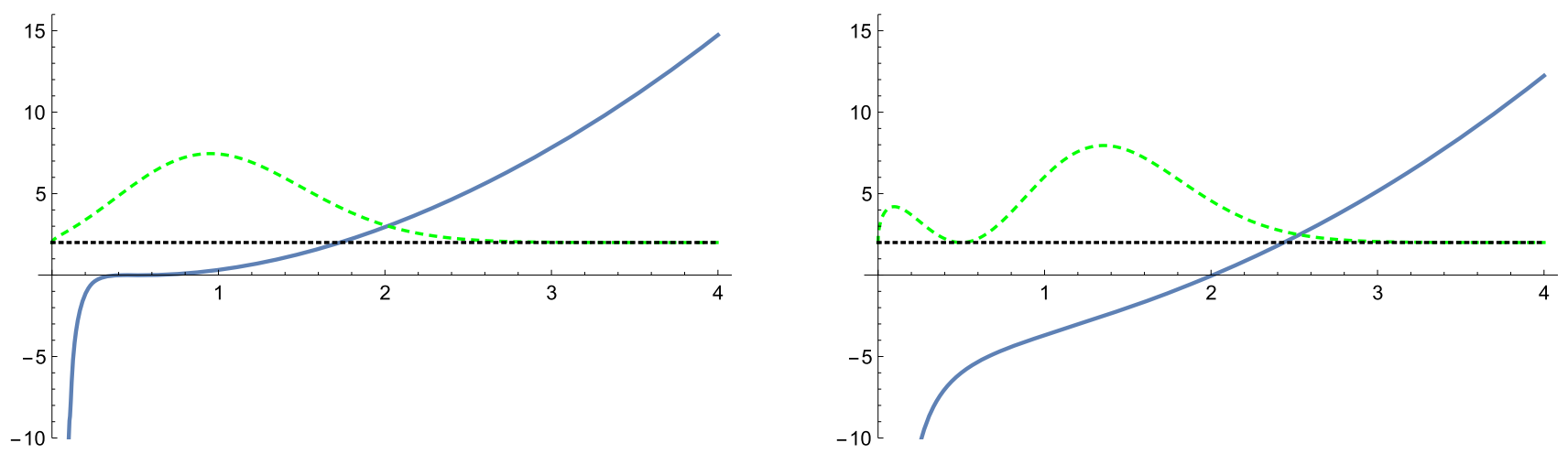

Fig. 3. The potential $\tilde{\tilde{V}}_{1}(x)$ (solid line) and probability density function $\left|\chi_{p}(x)\right|^{2}$ (dashed line) for $E_{1}=2+\sqrt{2}$ (left) and $E_{2}=2-\sqrt{2}$ (right) at the energy level $\varepsilon=2$.
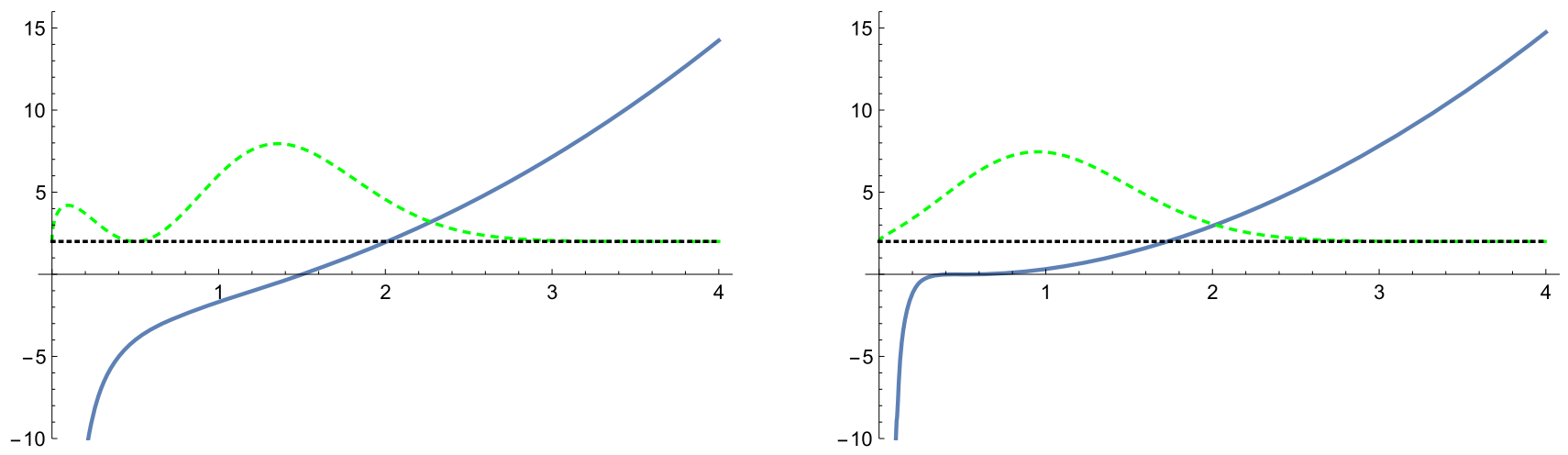

Fig. 4. The plots for case $t=-2 \sqrt{2} \rho$ of the potential $\tilde{\tilde{V}}_{1}(x)$ (solid line) and probability density function $\left|\chi_{p}(x)\right|^{2}$ (dashed line) for $E_{1}=2+\sqrt{2}$ (left) and $E_{2}=2-\sqrt{2}$ (right) at the energy level $\varepsilon=2$.

$\left|\psi_{p}\right\rangle=\gamma_{p, 1}|4,0\rangle+\gamma_{p, 2}|2,1\rangle+\gamma_{p, 3}|0,2\rangle, \quad p=1,2,3$,

with

\begin{tabular}{|r|r|r|r|r|}
\hline$p$ & $E_{p}$ & $\gamma_{p, 1}$ & $\gamma_{p, 2}$ & $\gamma_{p, 3}$ \\
\hline 1 & 8 & $\sqrt{3} /(2 \sqrt{2})$ & $1 / \sqrt{2}$ & $1 /(2 \sqrt{2})$ \\
\hline 2 & 0 & $-\sqrt{3} /(2 \sqrt{2})$ & $1 / \sqrt{2}$ & $-1 /(2 \sqrt{2})$ \\
\hline 3 & 4 & $1 / 2$ & 0 & $-\sqrt{3} / 2$ \\
\hline
\end{tabular}

We can write these eigenfunctions in terms of the variables $\rho, s$ as follows:

$\Psi_{p}(\rho, s)=s^{2}\left(\frac{1}{\sqrt{2}} \gamma_{p, 3}+2 \gamma_{p, 2} \rho+\frac{2 \sqrt{6}}{3} \gamma_{p, 1} \rho^{2}\right)$.

The wavefunctions are
$\chi_{p}(x)=e^{W(x)}\left(\frac{1}{\sqrt{2}} \gamma_{p, 3}+2 \gamma_{p, 2} \rho+\frac{2 \sqrt{6}}{3} \gamma_{p, 1} \rho^{2}\right)$.

When we choose $b=1$, that is, $x(\rho)=\rho$, we find the potential $\tilde{V}_{1}(x)$ and $\tilde{\tilde{V}}_{1}(x)$. The Schrödinger equation for both of these potentials has energy $E_{0}$. These potentials and the probability density functions $\left|\chi_{p}(x)\right|^{2}$ are shown in Fig. 5.

On the other hand, when we choose $b=1 / 2$, that is $x=\sqrt{\rho}$, we have Schrödinger equation with potential $\tilde{V}_{\frac{1}{2}}(x)$ as shown in (3.37) with the energy $\varepsilon_{p}=-2 \sqrt{2}\left(E_{p}\right)$. The potential $\tilde{V}_{\frac{1}{2}}(x)$ and the probability density function $\left|\chi_{p}(x)\right|^{2}$ can be seen in Fig. 6 (left). The same comments on the even eigenvalues and eigenfunctions apply in this example as in the previous $n=2$ and $b=1 / 2$ case. 

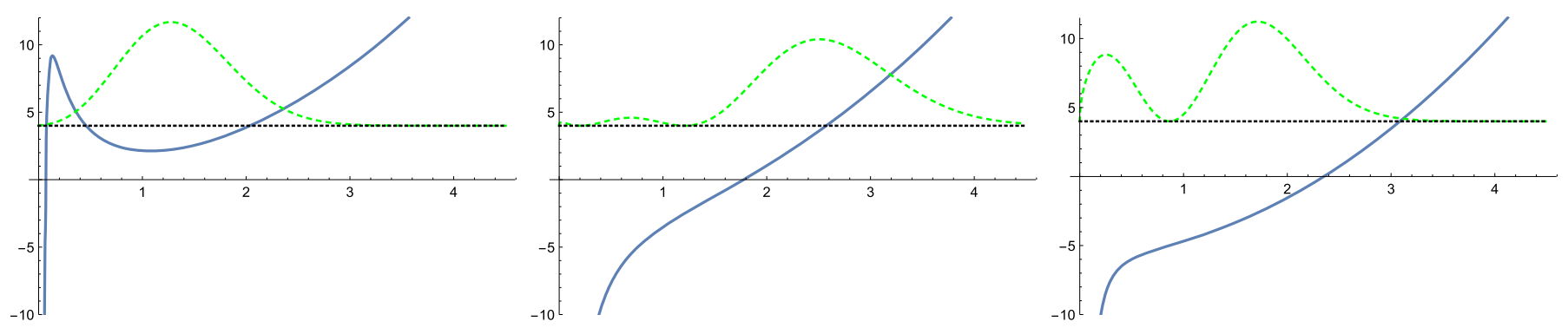

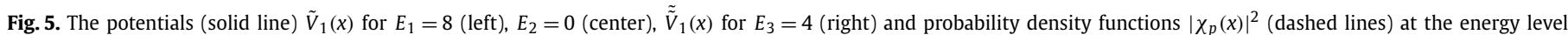
$\varepsilon=4$ (dotted line).
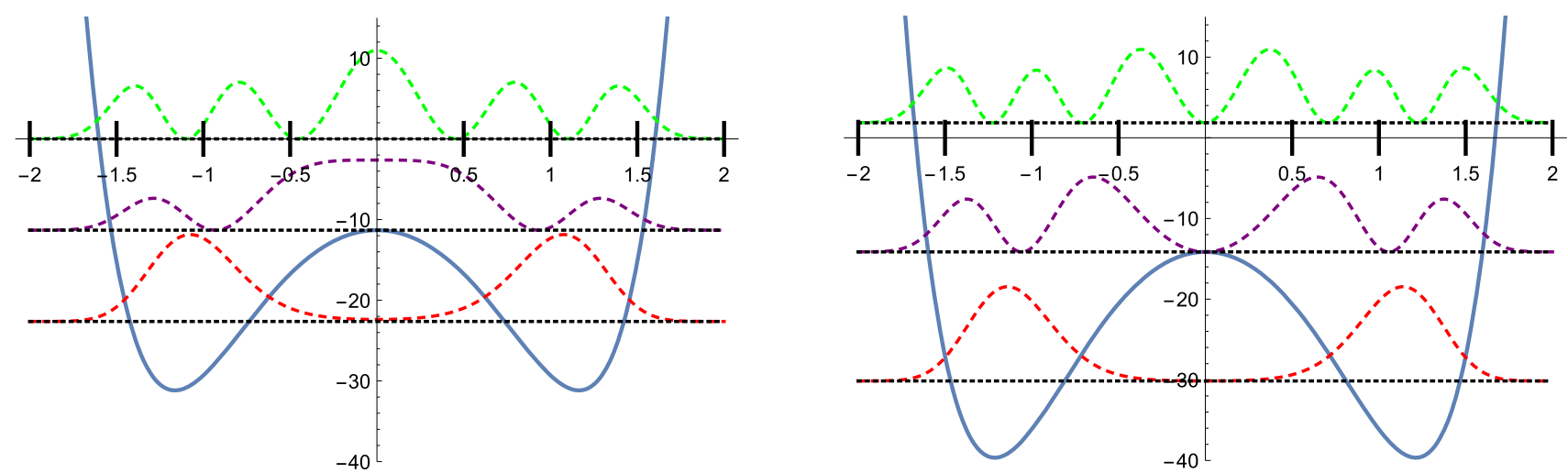

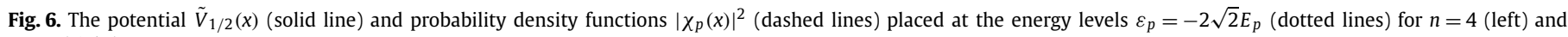
$n=5$ (right).

The wavefunctions for $n=5$ show the odd solutions for the potential $\tilde{V}_{\frac{1}{2}}(x)$ in this three-dimensional subspace. The eigenvalues and eigenfunctions in this subspace are

$\left|\psi_{p}\right\rangle=\gamma_{p, 1}|5,0\rangle+\gamma_{p, 2}|3,1\rangle+\gamma_{p, 3}|1,2\rangle, \quad p=1,2,3$,

with

\begin{tabular}{|r|r|r|r|r|}
\hline$p$ & $E_{p}$ & $\gamma_{p, 1}$ & $\gamma_{p, 2}$ & $\gamma_{p, 3}$ \\
\hline 1 & $5+4 \sqrt{2}$ & $\sqrt{5} / 4$ & $1 / \sqrt{2}$ & $\sqrt{3} / 4$ \\
\hline 2 & $5-4 \sqrt{2}$ & $-\sqrt{5} / 4$ & $1 / \sqrt{2}$ & $-\sqrt{3} / 4$ \\
\hline 3 & 5 & $\sqrt{3} / \sqrt{8}$ & 0 & $-\sqrt{5} / \sqrt{8}$ \\
\hline
\end{tabular}

After writing these eigenfunctions in terms of variables $(\rho, s)$, the wavefunctions will be in the following form:

$\chi_{p}(x)=e^{W(x)}\left(\frac{1}{\sqrt{2}} \gamma_{p, 3} x+\frac{2}{\sqrt{3}} \gamma_{p, 2} x^{3}+\frac{2 \sqrt{2}}{\sqrt{15}} \gamma_{p, 1} x^{5}\right)$.

In Fig. 6 , the potential $\tilde{V}_{1 / 2}(x)$ and probability density functions $\left|\chi_{p}(x)\right|^{2}$ are illustrated for $n=4$ and $n=5$.

\section{Conclusions}

In this work, in a first step we have studied the algebraic structure underlying the second harmonic generation Hamiltonian and later, as an application, we have developed some interesting differential realizations based on its symmetries. We began by writing the Hamiltonian of this system based on rising $J_{+}$and lowering $J_{\text {- }}$ the operators of the polynomial deformations of the Lie algebra $s l(2, R)$, using the finite dimensional space, with basis vectors $|J, M\rangle$, to calculate eigenvalue equation. Then we found a recurrence relation for the determinant of the matrix representation of the characteristic equation.
In deriving our results, we showed that we can predict the behavior of eigenvalues for the large number of photons. When the dimension of the subspace is even, the eigenvalues are in the form $E_{0} \pm g \sqrt{\xi_{i}}$. Also one of the eigenvalues will be zero for odddimensional subspace. On the other hand, we found that there is no radical expression for $n \geqslant 18$ which was obtained in [18].

The natural differential realization of the SHG Hamiltonian is obtained by means of the Bargmann-Fock representation. The change of variables to the new set of variables by using symmetry enables us to find the bi-confluent Heun equation as differential form of this system. The transformation between bi-confluent Heun equation and Schrödinger equation has been considered in many Refs. [35-39]. We made the map from this second-order differential equation to Schrödinger equation by a change of variable in the form $x=\rho^{b}$ and we have different set of quasi-exactly solvable potentials for different values of $b$.

As examples, we provided some explicit expressions for potential and wavefunctions for two values of $b=1$ and $b=1 / 2$ in two and three dimensional subspaces. We consider $\tilde{V}_{1}(x)$ and $\tilde{\tilde{V}}_{1}(x)$, $\tilde{V}_{1 / 2}(x)$ for $n=2,3$ and $n=4,5$ which have two and three dimensional subspace respectively. The potential $\tilde{V}_{1 / 2}(x)$ is a sextic potential which was obtained for this optical system in Refs. [27,40], while the other potential $\tilde{\tilde{V}}_{1}(x)$ is used to describe quantum dots. In two dimensional subspace, we have only one sextic potential for two different eigenfunctions for $b=1 / 2$. We have found even eigenfunctions that correspond to ground state and second excited state for $n=2$ in Fig. 1 and odd solutions that correspond to first and third excited state for $n=3$ in Fig. 2. On the other hand, for $b=1$, we have one potential $\tilde{\tilde{V}}_{1}(x)$ for each eigenfunctions. These eigenfunctions correspond to ground and first excited state which both of them have energy $\varepsilon=2$.

In second case, $n=4$, we have similar considerations. For $b=$ $1 / 2$, one sextic potential corresponds to three eigenfunctions with negative energies. For $n=4$, we have seen even eigenfunctions 
correspond to ground, second and fourth excited states and odd eigenfunctions correspond to first, third and fifth excited states for $n=5$ in Fig. 6 . For $b=1$, we have one potential for each of three eigenfunctions. These eigenfunctions correspond to ground, first and second excited states which all of them have energy $\varepsilon=4$.

As a brief summary, we found a family of quasi-exactly solvable potentials, labeled by the parameter $b$, which are related to the second harmonic generation. The two special values $b=1$ and $b=$ $1 / 2$ have been considered in detail. For $b=1 / 2$ we have found one potential with the first $n$ even eigenvalues. In the case $b=1$ for each $n \in \mathbb{N}$, we have found one eigenfunction (starting from the first up to the $n$-th) of $n$ different potentials.

\section{Declaration of competing interest}

The authors declare that they have no known competing financial interests or personal relationships that could have appeared to influence the work reported in this paper.

\section{Acknowledgements}

We would also like to show our gratitude to Prof. L.M. Nieto for his valuable comments during the course of this research and to the anonymous referee for constructive and notable comments which improved this work. Prof. Negro acknowledges partial financial support by the Spanish MINECO (MTM2014-57129-C2-1-P) and Junta de Castilla y León (BU229P18, VA137G18).

\section{References}

[1] F. Träger, Springer Handbook of Lasers and Optics, Springer Science \& Business Media, 2012

[2] P.A. Franken, A.E. Hill, C.W. Peters, G. Weinreich, Generation of optical harmonics, Phys. Rev. Lett. 7 (1961) 118.

[3] J.A. Armstrong, N. Bloembergen, J. Ducuing, P.S. Pershan, Interactions between light waves in a nonlinear dielectric, Phys. Rev. 127 (1962) 1918.

[4] D. Walls, Quantum theory of nonlinear optical phenomena, J. Phys. A, Gen. Phys. 4 (1971) 813.

[5] W.H. Louisell, A. Yariv, A.E. Siegman, Quantum fluctuations and noise in parametric processes. I, Phys. Rev. 124 (1961) 1646

[6] B.R. Mollow, R.J. Glauber, Quantum theory of parametric amplification. I, Phys. Rev. 160 (1967) 1076

[7] J. Tucker, D.F. Walls, Quantum theory of parametric frequency conversion, Ann. Phys. 52 (1969) 1.

[8] M. Kozierowski, R. Tanaś, Quantum fluctuations in second-harmonic light generation, Opt. Commun. 21 (1977) 229.

[9] P. Drummond, K. McNeil, D. Walls, Bistability and photon antibunching in sub/second harmonic generation, Opt. Commun. 28 (1979) 255.

[10] L. Mandel, Squeezing and photon antibunching in harmonic generation, Opt. Commun. 42 (1982) 437

[11] M. Hillery, Squeezing of the square of the field amplitude in second harmonic generation, Opt. Commun. 62 (1987) 135

[12] G. Drobný, I. Jex, Quantum properties of field modes in trilinear optical processes, Phys. Rev. A 46 (1992) 499.

[13] M. Kozierowski, S.M. Chumakov, Photon statistics in spontaneous emission fo the Dicke model in a lossless cavity and the generation of the Fock state, Phys. Rev. A 52 (1995) 4194
[14] E. Marcellina, J. Corney, M. Olsen, Improved quantum correlations in second harmonic generation with a squeezed pump, Opt. Commun. 309 (2013) 9.

[15] J. Shu, Spin-squeezing entanglement of second-harmonic generation, Int. J. Theor. Phys. 55 (2016) 4292.

[16] G. Alvarez, R.F. Alvarez-Estrada, Semiclassical analysis of a quasi-exactly solvable system: second harmonic generation, J. Phys. A, Math. Gen. 28 (1995) 5767.

[17] R. Alvarez-Estrada, A.G. Nicola, L. Sánchez-Soto, A. Luis, A quasiclassical analysis of second-harmonic generation, J. Phys. A, Math. Gen. 28 (1995) 3439.

[18] Y. Wu, X.-X. Yang, Spectrum and eigenstates for a model describing optical second harmonic generation, J. Opt. B, Quantum Semiclass. Opt. 5 (2003) 414.

[19] P.W. Higgs, Dynamical symmetries in a spherical geometry. I, J. Phys. A, Math. Gen. 12 (1979) 309.

[20] E.K. Sklyanin, Some algebraic structures connected with the Yang-Baxter equation, Funct. Anal. Appl. 16 (1982) 263.

[21] V. Karassiov, New lie-algebraic structures in nonlinear problems of quantum optics and laser physics, J. Sov. Laser Res. 13 (1992) 188

[22] V.P. Karassiov, A.B. Klimov, An algebraic approach to solving evolution problems in some nonlinear quantum models, Phys. Lett. A 189 (1994) 43.

[23] A. Klimov, J. Romero, J. Delgado, L. Sánchez-Soto, Master equations for effective Hamiltonians, J. Opt. B, Quantum Semiclass. Opt. 5 (2002) 34.

[24] V. Karassiov, A. Gusev, S. Vinitsky, Polynomial Lie algebra methods in solving the second-harmonic generation model: some exact and approximate calculations, Phys. Lett. A 295 (2002) 247.

[25] W. Miller Jr, S. Post, P. Winternitz, Classical and quantum superintegrability with applications, J. Phys. A, Math. Theor. 46 (2013) 423001.

[26] G. Álvarez, F. Finkel, A. González-López, M. Rodríguez, Quasi-exactly solvable models in nonlinear optics, J. Phys. A, Math. Gen. 35 (2002) 8705.

[27] N. Debergh, The relation between polynomial deformations of $\operatorname{sl}(2, R)$ and quasi-exact solvability, J. Phys. A, Math. Gen. 33 (2000) 7109.

[28] F. Caruso, J. Martins, V. Oguri, Solving a two-electron quantum dot model in terms of polynomial solutions of a biconfluent Heun equation, Ann. Phys. 347 (2014) 130.

[29] D. Gómez-Ullate, N. Kamran, R. Milson, Quasi-exact solvability and the direct approach to invariant subspaces, J. Phys. A, Math. Gen. 38 (2005) 2005.

[30] J.P. Tignol, Galois' Theory of Algebraic Equations, World Scientific Publishing Company, 2015.

[31] A. Ronveaux (Ed.), Heun's Differential Equations, Oxford University Press, Oxford, 1995.

[32] S.M. Reimann, M. Manninen, Electronic structure of quantum dots, Rev. Mod. Phys. 74 (2002) 1283.

[33] M. Taut, H. Eschrig, Exact solutions for a two-electron quantum dot model in a magnetic field and application to more complex systems, Z. Phys. Chem. 224 (2010) 631.

[34] J.S. Ardenghi, M. Gadella, J. Negro, Approximate solutions to the quantum problem of two opposite charges in a constant magnetic field, Phys. Lett. A 380 (2016) 1817.

[35] A. Ishkhanyan, Exact solution of the Schrödinger equation for the inverse square root potential $V_{0} / \sqrt{x}$, Europhys. Lett. 112 (2015) 10006.

[36] A. Ishkhanyan, A singular Lambert-W Schrödinger potential exactly solvable in terms of the confluent hypergeometric functions, Mod. Phys. Lett. A 31 (2016) 1650177.

[37] T. Ishkhanyan, A. Ishkhanyan, Solutions of the bi-confluent Heun equation in terms of the Hermite functions, Ann. Phys. 383 (2017) 79

[38] H. Sobhani, A. Ikot, H. Hassanabadi, Analytical solution of Bohr Hamiltonian and extended form of sextic potential using bi-confluent Heun functions, Eur. Phys. J. Plus 132 (2017) 240.

[39] H. Sobhani, H. Hassanabadi, W.S. Chung, Investigation of Bohr Hamiltonian in presence of Killingbeck potential using bi-confluent Heun functions, Nucl. Phys. A 973 (2018) 33.

[40] Y.-H. Lee, W.-L. Yang, Y.-Z. Zhang, Polynomial algebras and exact solutions of general quantum nonlinear optical models I: two-mode boson systems, J. Phys. A, Math. Theor. 43 (2010) 185204 